\title{
Indicação da terapia de reidratação oral no Setor de Emergência: decisão baseada na clínica?
}

\author{
Use of oral rehydration therapy in the Emergency Unit: a clinical-based decision?
}

\author{
Auxiliadora Damianne P. V. da Costa ${ }^{1}$, Gisélia Alves P. da Silva ${ }^{2}$
}

\section{RESUMO}

Objetivo: Revisar a literatura acerca da indicação da terapia de reidratação oral (TRO) no contexto do Setor de Emergência, buscando fatores inerentes à formação do médico, à atitude do cuidador e, finalmente, à dinâmica do próprio serviço como determinantes à sua aplicação.

Fontes de dados: Revisão não-sistemática da literatura incluindo artigos originais e meta-análises, nos idiomas inglês, português e espanhol, a partir das bases de dados Pubmed/Medline, Cochrane Collaboration, Lilacs e SciELO, no período de 1990 a 2008. Foram utilizados os termos "oral rehydration therapy", "diarrhea case management", "emergency department" e palavras relacionadas.

Síntese dos dados: Realizada em local apropriado, a TRO mostrou eficácia semelhante à terapia venosa no restabelecimento do nível de hidratação em crianças com diarreia aguda no Setor de Emergência. O tempo de formado e a experiência profissional, o conhecimento e o treinamento no manejo da diarreia aguda mostraram associação à utilização da TRO. Entretanto, relatos de inconveniência de sua administração no Setor de Emergência incluem falta de espaço físico e pressão assistencial, sugerindo, ao mesmo tempo, inadequação estrutural e uso inapropriado do serviço nesses casos. A relação com o cuidador também influencia na decisão médica, com o relato de desconfiança deste quanto à eficácia da terapia sendo citado como barreira à sua indicação.

Conclusões: A subutilização da TRO no Setor de Emergência está associada a fatores extrínsecos à formação médica, como questões estruturais e fatores inerentes à relação com o cuidador diante das suas expectativas quanto à terapia.
Palavras-chave: serviços médicos de emergência; terapia de reidratação oral; gastroenterite; criança.

\section{ABSTRACT}

Objective: To review the literature about indication of oral rehydration therapy (ORT) for children in the emergency unit, seeking factors related to medical training, caregiver's attitude and units' conditions as determinants for that practice.

Data sources: Non-systematic literature review including original articles and meta-analysis in English, Portuguese and Spanish, identified via Pubmed/Medline, Cochrane Collaboration, Lilacs and SciELO, published between 1990 and 2008, using the terms "oral rehydration therapy", "diarrhea case management", "emergency department" and related words.

Data synthesis: When carried out in appropriate rooms, ORT treatment is as effective as intravenous therapy in restoring the level of hydration in children with acute diarrhea in the emergency unit. Time of graduation and professional experience, knowledge and training on acute diarrhea case management showed an association with ORT use. However, reports of inconvenience of its administration in the emergency unit include lack of physical space and pressure care, while simultaneously suggesting structural inadequacy and improper use of the service in these cases. Reports of caregivers' suspicion about the effectiveness of this therapy are cited as a barrier for prescription.

Conclusions: Subuse of ORT in the emergency unit is associated with factors beyond medical training and exper-
Instituição: Universidade Federal de Pernambuco (UFPE), Recife, PE, Brasil

${ }^{1}$ Mestre; Preceptora do Setor de Emergência Pediátrica do Instituto de Medicina Integral Prof. Fernando Figueira (IMIP), Recife, PE, Brasil

2Doutora; Professora do Departamento Materno-Infantil da UFPE, Recife, PE, Brasil
Endereço para correspondência:

Auxiliadora Damianne P. V. da Costa

Rua Antônio Valdevino Costa, 280, bloco 4, apto. 501 -

CEP 50640-040 - Recife/PE

E-mail: doradami@gmail.com

Conflito de interesse: nada a declarar

Recebido em: 15/7/09

Aprovado em: 1/12/09 
tise, such as structural problems and caregivers' expectations about the therapy.

Key-words: emergency medical services; oral rehydration therapy; gastroenteritis; child.

\section{Introdução}

Poucas descobertas tiveram tanto impacto na redução da gravidade e letalidade de uma afecção como a introdução da terapia de reidratação oral (TRO) no manejo da doença diarreica aguda no final da década de 1970. Mesmo permanecendo como causa importante de morbidade em crianças menores de cinco anos - com média de dois a três episódios por criança ao ano - a diarreia aguda experimentou uma queda significativa em sua mortalidade anual nessa faixa etária: da elevada cifra de 4,6 milhões, em 1980, para 3,3 milhões em 1990 e, finalmente, situando-se entre 1,5 e 2,5 milhões em $1999^{(1-5)}$. Indicada para crianças com desidratação leve a moderada, a TRO é considerada solução simples, de baixo custo, praticamente isenta de complicações e menos traumática para a criança, com eficácia semelhante à terapia de reidratação venosa $(\mathrm{TRV})^{(5-10)}$.

No Setor de Emergência, entretanto, relatos médicos revelam preferência pela terapia venosa em cenários de desidratação não-grave por diarreia aguda ${ }^{(6,11-14)}$. As justificativas incluem questões associadas ao suporte estrutural (falta de espaço físico apropriado e de recursos humanos treinados), inerentes à atitude do cuidador (preferência pela TRV) e dúvidas relacionadas à real eficácia da TRO para o setor (crença numa maior demanda de tempo requerida por esta em relação à TRV e maior falha terapêutica nos casos com desidratação moderada e/ou vômitos) ${ }^{(11-14)}$. A demanda excessiva da assistência também é citada como uma barreira à indicação da $\mathrm{TRO}^{(14)}$, com possível uso inapropriado do setor.

O estudo da aplicação de uma prática em saúde num setor específico permite aferir simultaneamente a qualidade da assistência prestada e a adequação dessa mesma prática à função desse serviço. O objetivo desta revisão é situar a TRO no contexto do Setor de Emergência, buscando fatores inerentes à formação do médico que atua no setor, à atitude do cuidador e, finalmente, à dinâmica do próprio serviço como determinantes à sua aplicação.

\section{Fontes dos dados}

Foram utilizadas as bases de dados Pubmed/Medline, Cochrane Collaboration, Lilacs e SciELO, com busca de artigos originais e meta-análises relacionados aos seguintes tópicos:
1. Eficácia da TRO no Setor de Emergência.

2. Atitude do cuidador, relacionada à TRO e ao uso apropriado do Setor de Emergência.

3. Estrutura do setor (adequação à prestação do serviço e função na rede de assistência).

4) Perfil e formação profissional do médico que atua no setor.

A combinação de termos utilizada foi:

1. Oral rehydration therapy or diarrhea case management and emergency department, knowledge, attitude, training, practice.

2. Emergency department and quality, overcrowding, non-urgent visits, primary care, inappropriate use, burnout.

A busca abrangeu o período de 1990 a 2008, com seleção de textos completos publicados nos idiomas inglês, português e espanhol.

\section{Síntese dos dados}

\section{A eficácia da TRO no Setor de Emergência}

Algumas situações clínicas são apontadas como empecilhos à execução da TRO no Setor de Emergência. A presença ou intensidade dos vômitos e a desidratação moderada estão entre elas $^{(11-14)}$. Em dois ensaios clínicos randomizados conduzidos especificamente em crianças com desidratação moderada e com técnicas de alocação adequadas, não houve diferença entre os grupos submetidos à TRO ou TRV para o restabelecimento do déficit hidroeletrolítico ${ }^{(15,16)}$. O pequeno número de participantes em cada grupo de intervenção (TRO ou TRV) é uma limitação dos estudos, mas os grupos são bastante homogêneos em relação aos escores de desidratação (foram utilizadas escalas para classificar o estado de hidratação).

Quanto aos vômitos, apesar de haver maiores taxas de falha terapêutica nos estudos que não excluem pacientes nos quais eles são persistentes antes da alocação para o tipo de hidratação, essa variável não apresenta significância estatística após metanálise ${ }^{(10)}$. A administração de volumes menores em intervalos mais curtos frequentemente diminui sua incidência, permitindo a manutenção da $\mathrm{TRO}^{(8)}$.

A maior demanda de tempo requerida em relação à terapia venosa é outro inconveniente citado para administrar TRO no Setor de Emergência que não se confirma na literatura ${ }^{(9,16-}$ ${ }^{19)}$. Pode ser avaliada de duas formas: tempo de permanência no Setor de Emergência e no hospital (que inclui também o tempo de internação). Em relação ao tempo de permanência hospitalar, foi demonstrado recentemente, em duas meta- 
análises, tendência à sua redução quando a terapia oral é utilizada $^{(9,10)}$. Estudo piloto desenvolvido em emergência pediátrica australiana avaliou as taxas de admissão hospitalar, o número de retornos não-agendados e o tempo total de permanência hospitalar e no Setor de Emergência antes e após a implantação de um protocolo de TRO para crianças com diarreia aguda ${ }^{(18)}$. Especificamente em relação aos dois últimos desfechos, observou-se redução da permanência total no hospital, sem alteração do tempo de observação no Setor de Emergência ${ }^{(18)}$. A redução no tempo de permanência hospitalar pode estar relacionada, ao menos em parte, ao menor número de admissões hospitalares observadas quando se utiliza a $\mathrm{TRO}^{(18)}$. Quanto à permanência semelhante observada com os dois tipos de terapia na emergência, a explicação poderia estar no fato de, apesar de a reposição do déficit com a TRO poder exigir um tempo maior, o início é mais rápido (não necessita de procedimento invasivo) e a realimentação mais precoce $e^{(\mathfrak{l}, 16)}$.

Existem algumas limitações metodológicas nos estudos de eficácia da TRO disponíveis, como a dificuldade na avaliação cega (pela própria natureza da intervenção - oral versus venosa), pequeno número de participantes, heterogeneidade em relação ao tipo de solução oral empregada, idade dos participantes e falta de padronização quanto ao grau de desidratação ${ }^{(9,10)}$. No geral, entretanto, demonstrase de eficácia igual ou mesmo superior da terapia oral em relação à venosa no restabelecimento do déficit para os casos de desidratação não-grave $e^{(9,10,15-18,20)}$.

\section{O Setor de Emergência}

Nos estudos de eficácia da TRO no Setor de Emergência, a intervenção (TRO ou TRV) é realizada em salas específicas, sob a supervisão de profissionais de saúde habilitados ${ }^{(15,16,18)}$. A presença de uma sala de reidratação oral, entretanto, não é uma realidade na maioria dos serviços de emergência. Assim, além da qualidade do atendimento, a realização da TRO nesse setor levanta a questão da função assistencial do serviço.

Num sistema hierarquizado de saúde, o papel do Setor de Emergência é o de oferecer serviço adequado a pacientes com condições que implicam risco de morte ou perda de função, exigindo tratamento imediato. Definição proposta pelo American College of Emergency Physicians (ACEP), em 1994, considera como condição médica de emergência aquela de aparecimento recente e suficientemente grave (incluindo dor importante) que leva o indivíduo a acreditar que a ausência de tratamento imediato poderia resultar em grave prejuízo de sua saúde ou de funções orgânicas ${ }^{(21)}$.
A diarreia aguda é causa frequente de busca por atendimento de urgência ${ }^{(22,23)}$. Complicada com desidratação grave, configura condição de emergência, uma vez que pode levar, a choque hipovolêmico e mesmo ao óbito em poucas horas na ausência de intervenção adequada (hidratação venosa), principalmente nas crianças de baixa idade ${ }^{(8)}$. Na maioria dos casos, entretanto, não há desidratação ou a condição é leve a moderada, situação em que o tratamento preconizado é a $\mathrm{TRO}^{(7)}$. Como terapia oral e sem sofisticação, a TRO pode ser conduzida em nível primário, uma vez que as expectativas para o Setor de Emergência são de um atendimento rápido, de alta tecnologia e complexidade ${ }^{(24)}$.

Casos de diarreia aguda que implicam administração da TRO configuram um exemplo de uso inapropriado dos serviços de emergência, inadequado para a complexidade do tipo de serviço oferecido. Aliada a problemas estruturais, esse tipo de demanda resulta em sobrecarga das unidades de emergência, gerando um cenário que acaba por prejudicar o atendimento dos casos que necessitam realmente de tratamento de urgência ${ }^{(24)}$. É uma situação encontrada particularmente em países em desenvolvimento, onde obstáculos à efetivação de serviços incluem, além da sustentabilidade face à grande demanda, problemas com custos, falta de modelos estruturados e de treinamento apropriado dos profissionais envolvidos ${ }^{(25)}$.

\section{A atitude do cuidador}

A falta de uma definição padronizada de "urgência" e "emergência" gera conflitos entre profissionais de saúde e pacientes na hora do atendimento e, principalmente, na elaboração de sistemas de triagem, afetando particularmente aqueles que lidam com pacientes mais jovens, nos quais as peculiaridades conferidas pela baixa idade podem atribuir gravidade a casos aparentemente não-graves ${ }^{(26)}$. Esforço observado na busca desta definição e, portanto, de sistemas de triagem adequados coincide com o grande aumento ocorrido, nos últimos anos, na demanda pelo Setor de Emergência, principalmente no que tange às chamadas condições não-urgentes. Com frequência variável nos diversos estudos, de acordo com o método utilizado e definições de urgência e emergência empregadas, cerca de 20 a 66\% dos pacientes que se apresentam ao setor o fazem por causas não-urgentes, podendo ser conduzidos, a princípio, em nível primário de saúde ou em unidades menores de atendimento imediato $^{(22,24,27-34)}$

O perfil desses pacientes com causas não-urgentes mostra algumas semelhanças entre os estudos: são mais jovens, do 
sexo feminino, apresentando consultas que raramente resultam em internação, não referenciados por médico e com sintomas de longa duraçãa ${ }^{(22,29,31,35,36)}$. Alguns (numa frequência bastante variável de 22 a $82 \%$ ) acreditam realmente, no momento da consulta, que sua condição (ou da criança por quem é responsável) configura uma emergência ${ }^{(31,35,37,38)}$. Mas várias outras razões estão implicadas: insatisfação com o atendimento em nível primário, acessibilidade (proximidade, dificuldade de acesso ao médico assistente ou consulta em nível primário), possibilidade de diagnóstico imediato, tratamento adequado e confiança no serviço de emergência ${ }^{(24,27,34-39)}$.

Duas questões, portanto, parecem nortear a busca inadequada pelo Setor de Emergência: uma educacional (que envolve discernimento sobre o conceito de emergência e funções dos níveis de assistência) e outra relacionada a uma aparente insuficiência da atenção básica em fornecer assistência adequada. Assim, melhora do acesso e da qualidade dos cuidados primários e secundários, bem como da relação entre os diferentes níveis de assistência, aliada a campanhas educacionais junto à população sobre o uso apropriado dos Serviços de Saúde poderiam ser a solução do problema ${ }^{(34)}$. A qualidade da assistência e a satisfação do usuário no nível primário demonstraram reduzir em 35 a $50 \%$ as visitas ao Setor de Emergência por causas não-urgentes ${ }^{(40)}$. Projetos de implantação de sistemas de referência a partir desse setor para a atenção básica (redirecionamento), alguns com reforço coordenado nesse nível, foram efetivos em reduzir o número de visitas à emergência por causas não-urgentes ${ }^{(41,42)}$.

Além do uso inapropriado do serviço (a maioria dos casos pode ser conduzida em nível primário), a abordagem do manejo da doença diarreica aguda no Setor de Emergência envolve outro aspecto importante: as expectativas do cuidador sobre a terapia. Essa interação com o responsável pela criança é uma particularidade inerente ao tratamento do paciente pediátrico. Nesse sentido, a perspectiva do cuidador sobre a terapia é relevante para a decisão médica, com a desconfiança dos pais ou responsáveis com relação à sua eficácia sendo apontada como inconveniente à administração da TRO ${ }^{(14)}$.

Essa ideia de preferência pela hidratação venosa, entretanto, não se confirma em ensaios clínicos randomizados, havendo grau de satisfação com a TRO semelhante ou mesmo superior em relação à terapia venosa na entrevista de seguimento com pais e responsáveis após o atendimento em emergência de crianças com desidratação não-grave $e^{(9,15,16)}$. Sua efetividade, somada ao fato de ser menos traumática para a criança (não envolve procedimento invasivo), pode justificar tal achado.

\section{O médico do Setor de Emergência: perfil e formação profissional}

Responsável pela indicação da terapia, o médico tem sua decisão norteada pelo conhecimento das diretrizes e normas vigentes sobre o manejo da doença e sua motivação para a aplicação da mesma. Esta última encontra-se permeada por fatores extrínsecos, que incluem os determinantes estruturais e associados à relação com o binômio cuidador-criança.

A associação entre maior grau de conhecimento sobre a TRO e sua utilização ainda é bastante controversa ${ }^{(11-13)}$. Em estudo norte-americano, no qual apenas $37 \%$ dos pediatras entrevistados eram realmente familiarizados com as recomendações da Academia Americana de Pediatria (AAP) para o manejo da desidratação por diarreia, o grupo dito "familiarizado" com tais recomendações referiu mais frequentemente o uso da TRO que o grupo "não-familiarizado", em situações de desidratação leve (81 versus 66\%) e moderada (25 versus $10 \%)^{(13)}$.

O tempo de graduação também parece influenciar no uso da TRO para cenários de desidratação leve e moderada. Outro estudo realizado também entre membros da AAP com área de atuação em emergência revelou diferenças na sua indicação entre recém-graduados e pediatras com maior tempo de formação. Enquanto $95 \%$ dos casos de desidratação leve e $55 \%$ dos desidratados moderados são conduzidos com TRO entre os recém-graduados, essas frequências situamse entre 86 e $33 \%$, respectivamente, no grupo de médicos com maior tempo de formação ${ }^{(19)}$. O conhecimento sobre a refutação da ideia de que a TRO implica maior tempo no Setor de Emergência para a reidratação, entretanto, foi baixo e semelhante entre os dois grupos (19 versus 24\%). Além disso, estudos de avaliação de serviço não demonstraram incremento significativo na frequência de sua indicação após treinamento em unidades para o manejo da diarreia ${ }^{(43-46)}$. Houve desproporção entre o grande aumento observado no conhecimento, avaliado por meio de testes específicos, e o modesto incremento na utilização da $\mathrm{TRO}^{(43)}$. Esses dados sugerem que a indicação da terapia envolve fatores que estão além do treinamento e conhecimento de sua eficácia.

A sobrecarga nas salas emergência decorrente da demanda excessiva se reflete na insuficiência de materiais e insumos e afeta particularmente o profissional que atua nesse setor. Tal cenário vai de encontro ao próprio discurso de humanização do atendimento que, além do objetivo de satisfação do usuário, inclui o fornecimento de adequadas condições de trabalho ao profissional que presta esse serviço nas várias áreas de assistência ${ }^{(47)}$. 
Mais especificamente em relação às condições de trabalho, estudos recentes revelam a questão do burnout do profissional de saúde, particularmente aquele que trabalha em emergência. São componentes associados a essa síndrome a exaustão emocional, a falta de realização profissional e a despersonalização ${ }^{(48,49)}$. Os relatos desses profissionais revelam sentimentos de cansaço, angústia, revolta pela sobrecarga de trabalho (frequentemente mal remunerada) e limitações de recursos diante das situações de estresse associadas ao risco de vida $^{(49)}$. Inquérito nacional realizado recentemente pela Sociedade Brasileira de Pediatria juntamente com a Fundação Oswaldo Cruz revelou que quase $80 \%$ dos pediatras trabalham em regime de plantão e $78 \%$ afirmam possuir sinais de desgaste profissional ${ }^{(50)}$.

Esse desgaste físico e psíquico, resultado das situações de estresse peculiares à profissão e aliado à pressão exercida pela demanda excessiva da assistência, participa como agente modificador da atitude médica, nas relações de trabalho e na interação com o paciente e seu cuidador. Os relatos de inconveniência de administração da TRO na prática, percep-

\section{Referências bibliográficas}

1. Victora CG, Bryce J, Fontaine O, Monasch R. Reducing deaths from diarrhoea through oral rehydration therapy. Bull World Health Organ 2000;78: 1246-55.

2. Kosek M, Bern C, Guerrant RL. The global burden of diarrhoeal disease, as estimated from studies published between 1992 and 2000. Bull World Health Organ 2003;81:197-204.

3. Thapar N, Sanderson IR. Diarrhoea in children: an interface between developing and developed countries. Lancet 2004;363:641-53.

4. Sur D, Bhattacharya SK. Acute diarrhoeal diseases--an approach to management. J Indian Med Assoc 2006;104:220-3.

5. Forsberg BC, Petzold MG, Tomson G, Allebeck P. Diarrhoea case management in low- and middle-income countries-an unfinished agenda. Bull World Health Organ 2007;85:42-8.

6. King CK, Glass R, Bresee JS, Duggan C; Centers for Disease Control and Prevention. Managing acute gastroenteritis among children: oral rehydration, maintenance and nutritional therapy. MMWR Recomm Rep 2003;52:1-16.

7. World Health Organization [homepage on the Internet]. The treatment of diarrhea: a manual for physicians and other senior health workers [cited 2009 Feb 18]. Available from: http://whqlibdoc.who.int/publications/2005/9241593180.pdf

8. [No authors listed]. Practice parameter: the management of acute gastroenteritis in young children. Pediatrics 1996;97:424-36.

9. Fonseca BK, Holdgate A, Craig JC. Enteral vs intravenous rehydration therapy for children with gastroenteritis: a meta-analysis of randomized controlled trials. Arch Pediatr Adolesc Med 2004;158:483-90.

10. Hartling L, Bellemare S, Wiebe N, Russell K, Klassen TP, Craig W. Oral versus intravenous rehydration for treating dehydration due to gastroenteritis in children. Cochrane Database Syst Rev 2006;3:CD004390.

11. Reis EC, Goepp JG, Katz S, Santosham M. Barriers to use of oral rehydration therapy. Pediatrics 1994;93:708-11.

12. Conners GP, Barker WH, Mushlin AI, Goepp JG. Oral versus intravenous: rehydration preferences of pediatric emergency medicine fellowship directors. Pediatr Emerg Care 2000;16:335-8. ção de preferência da equipe pela terapia venosa e falta de treinamento dessa em seu manejo ${ }^{(11-14)}$ refletem tal dificuldade em lidar com os processos de trabalho no setor.

\section{Conclusões}

Concebida para ser realizada em unidades de reidratação oral, sob supervisão adequada, a TRO é uma atribuição do nível primário de assistência, com necessidade de reorientação dessa demanda dos serviços de emergência para a atenção básica. Tal redirecionamento pressupõe tanto o aspecto educacional do usuário (em relação às funções dos níveis de assistência) como a adequação estrutural da rede. A implantação de protocolos de reidratação oral (com salas específicas para esse fim), entretanto, é uma alternativa para uma melhor qualidade no atendimento desses pacientes que chegam ao Setor de Emergência, quando o nível primário falha em fornecer assistência adequada. Nesse sentido, além de suporte estrutural adequado, educação e treinamento médicos e da equipe de apoio são essenciais.

13. Ozuah PO, Avner JR, Stein RE. Oral rehydration, emergency physicians, and practice parameters: a national survey. Pediatrics 2002;109:259-61.

14. Sociedad Españhola de Urgencias de Pediatría. Utilización de la rehidratación oral en urgencias: encuesta nacional. An Pediatr (Barc) 2004;60: 243-8.

15. Atherly-Jonh YC, Cunningham SJ, Crain EF. A randomized trial of oral vs intravenous rehydration in a pediatric emergency department. Arch Pediatr Adolesc Med 2002;156:1240-3.

16. Spandorfer PR, Alessandrini EA, Joffe MD, Localio R, Shaw KN. Oral versus intravenous rehydration of moderately dehydrated children: a randomized, controlled trial. Pediatrics 2005;115:295-301.

17. Guarino A, Albano F, Ashkenazi S, Gendrel D, Hoekstra JH, Shamir R et al European Society for Paediatric Gastroenterology, Hepatology, and Nutritional / European Society for Paediatric Infectious Diseases evidence-based guidelines for the management of acute gastroenteritis in children in Europe. J Pediatr Gastroenterol Nutr 2008;46 (Suppl 2):S81-122.

18. Boyd R, Busuttil M, Stuart P. Pilot study of a paediatric emergency department oral rehydration protocol. Emerg Med J 2005;22:116-7.

19. Bender BJ, Ozuah PO, Crain EF. Oral rehydration therapy: is anyone drinking? Pediatr Emerg Care 2007;23:624-6.

20. Duggan C, Lasche J, McCarty M, Mitchell K, Dershewitz R, Lerman SJ et al Oral rehydration solution for acute diarrhea prevents subsequent unscheduled follow-up visits. Pediatrics 1999;104:e29.

21. Li J, Galvin HK, Johnson SC. The "prudent layperson" definition of an emergency medical condition. Am J Emerg Med 2002;20:10-3.

22. Pileggi C, Raffaele G, Angelillo IF. Paediatric utilization of an emergency department in Italy. Eur J Public Health 2006;16:565-9.

23. Armon K, Stephenson T, MacFaul R, Eccleston P, Werneke U. An evidence and consensus based guideline for acute diarrhoea management. Arch Dis Child 2001;85:132-42.

24. Kovacs MH, Feliciano KV, Sarinho SW, Veras AA. Access to basic care for children seen at emergency departments. J Pediatr (Rio J) 2005;81:251-8. 
25. Razzaq JA, Kellermann AL. Emergency medical care in developing countries: is it worthwhile? Bull World Health Organ 2002;80:900-5.

26. Melo EM, Assunção AA, Ferreira RA. Pediatric practice in public emergency services and factors affecting care. Cad Saude Publica 2007;23:3000-10.

27. Oktay C, Cete Y, Eray O, Pekdemir M, Gunerli A. Appropriateness of emergency department visits in a Turkish university hospital. Croat Med J 2003;44:585-91.

28. Oberlander TF, Pless IB, Dougherty GE. Advice seeking and appropriate use of a pediatric emergency department. Am J Dis Child 1993;147:863-7.

29. Lang T, Davido A, Diakité B, Agay E, Viel JF, Flicoteaux B. Non-urgent care in the hospital medical emergency department in France: how much and which health needs does it reflect? J Epidemiol Comm Health 1996;50: 456-62.

30. Coleman P, Irons R, Nicholl J. Will alternative immediate care services reduce demands for non-urgent treatment at accident and emergency? Emerg Med J 2001;18:482-7.

31. Bianco A, Pileggi C, Angelillo IF. Non-urgent visits to hospital emergency department in Italy. Public Heath 2003;117:250-5.

32. Suruda A, Burns TJ, Knight S, Dean JM. Health insurance, neighborhood income, and emergency department usage by Utah children 1996-1998. BMC Health Serv Res 2005;13;5:29.

33. Moon TD, Laurens MB, Weimer SM, Levy JA. Nonemergency emergency room utilization for an inner-city pediatric population. Pediatr Emerg Care 2005;21:363-6.

34. Carret ML, Fassa AG, Kawachi I. Demand for emergency health service: factors associated with inappropriate use. BMC Health Ser Res 2007;7:131.

35. Afilalo J, Marinovich A, Afilalo M, Colacone A, Léger R, Unger B et al. Nonurgent emergency department patient characteristics and barriers to primary care. Acad Emerg Med 2004;11:1302-10.

36. Ryan M, Spicer M, Hyett C, Barnett P. Non-urgent presentations to a paediatric emergency department: parental behaviours, expectations and outcomes. Emerg Med Australas 2005;17:457-62.

37. Truman CD, Reutter L. Care-giving and care-seeking behaviours of parents who take their children to an emergency department for non-urgent care. Can J Public Health 2002;93:41-6.
38. Doobinin KA, Heidt-Davis PE, Gross TK, Isaacman DJ. Nonurgent pediatric emergency department visits: Care-seeking behavior and parental knowledge of insurance. Pediatr Emerg Care 2003;19:10-4.

39. Woolfenden S, Ritchie J, Hanson R, Nossar V. Parental use of a paediatric emergency department as an ambulatory care service. Aust $\mathrm{N} Z \mathrm{~J}$ Public Health 2000;24:204-6.

40. Brousseau DC, Hoffmann RG, Nattinger AB, Flores G, Zhang Y, Gorelick M. Quality of primary care and subsequent pediatric emergency department utilization. Pediatrics 2007;119:1131-8.

41. Hansagi $H$, Allebeck $P$, Edhag $O$. Health care utilization after referral from a hospital emergency department. Scand J Soc Med 1989;17:291-9.

42. Wang C, Villar ME, Mulligan DA, Hansen T. Cost and utilization analysis of a pediatric emergency department diversion project. Pediatrics 2005;116:1075-9.

43. Salazar-Lindo E, Chea-Woo E, Kohatsu J, Miranda PR. Evaluation of clinical management training program for diarrhea. J Diarrhoeal Dis Res 1991;9: 227-34.

44. Baig LA, Thaver I. Does training affect quality of diarrhea case management? J Pak Med Assoc 1997;47:204-7.

45. Kundi MZ, Ahmad I, Anjum M. Evaluation of diarrhoea management of health professionals trained at Diarrhea Training Unit of Rawalpindi General Hospital. J Pak Med Assoc 1997;47:3-6.

46. Ibrahim S, Isani Z. Evaluation of doctors trained at Diarrhoea Training Unit of National Institute of Child Health, Karachi. J Pak Med Assoc 1997;47:7-11.

47. Oliveira $B R$, Collet $N$, Viera CS. A humanização na assistência à saúde. Rev Latino-Am Enfermagem 2006;14:277-84.

48. Albaladejo R, Villanueva R, Ortega P, Astasio P, Calle ME, Dominguez V. Síndrome de burnout en el personal de enfermería de un hospital de Madrid. Rev Esp Salud Publica 2004;78:505-16.

49. Feliciano KV, Kovacs MH, Sarinho SW. Feelings of paediatric emergency service professionals: reflexions on burnout. Rev Bras Saude Matern Infant 2005;5:319-28.

50. Machado MH, Vaz ES. Perfil dos pediatras no Brasil: relatório final. Rio de Janeiro: Sociedade Brasileira de Pediatria; 2001. 